\title{
Simulation of Ablation in a Sounding Rocket Thermal Protection System Via an Interface Tracking Method with Two Moving Fronts
}

\author{
Humberto Araujo Machado
}

\begin{abstract}
In this work, an interface tracking method is employed to simulate the ablative process in the region near the stagnation point of the VSB-30 sounding rocket Thermal Protection System (TPS). The ablation model considers the presence of two-fronts, the char-melting and the pyrolysis fronts. The results for the proposed model are compared to the ones obtained from the traditional one-front model, which supposes a constant ablation temperature. Results show that the one-front model overestimates the ablation period, mass loss and the internal temperature after the flight. The increase in the accuracy with this model shall provide a better dimensioning of the TPS, reducing its weight and cost.
\end{abstract}

KEYWORDS: TPS, ablation, moving boundary, computational simulation, sounding rocket.

\section{INTRODUCTION}

Sounding rockets are extensively used to provide microgravity environments for scientific experiments. The Institute of Aeronautics and Space (Instituto de Aeronáutica e Espaço - IAE) of Brazil has designed, built and launched hundreds of rockets along the past 40 years. The VSB-30 sounding rocket is a two-stage unguided solid propellant rocket used by the Brazilian Space Agency (AEB) and European Space Agency (ESA).

Figure 1 shows a schematic representation of VSB-30. It has a total length of $13 \mathrm{~m}$ and a diameter of $0.6 \mathrm{~m}$. It is equipped with two solid propellant motors, namely S31 and S30. S31 acts as a booster during its $15 \mathrm{~s}$ burning time, whereas $\mathrm{S} 30$ burns for about $30 \mathrm{~s}$, reaching an apogee of $250 \mathrm{~km}$, for a payload mass of $400 \mathrm{~kg}$. VSB-30 was developed by IAE in cooperation with the German Space Agency (DLR). So far, several VSB-30 successful flights occurred, from Alcântara Launch Center (CLA), in Brazil, and from Esrange, Sweden (Garcia et al., 2011), Fig. 2. During its flight, VSB-30 provides 6 minutes of microgravity environment. It is worth mentioning that an $\mathrm{S} 30$ motor developed and fabricated by IAE equipped the VS-30-Orion rocket used by Sharp Edge Flight Experiment (SHEFEX) (Turner et al., 2005).

Figure 3 presents the nominal altitude-velocity map for a VSB-30 atmospheric flight. According to Fig. 3, VSB-30

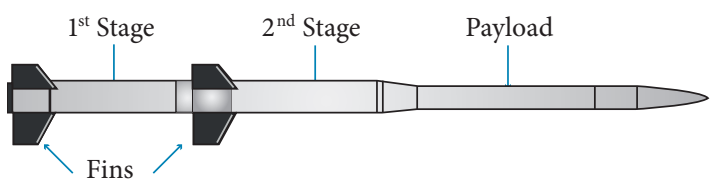

Figure 1. VSB-30 sounding rocket.

三 1. Instituto de Aeronáutica e Espaço - São José dos Campos/SP - Brazil

三 Author for correspondence: Humberto Araujo Machado | Praça Marechal Eduardo Gomes, 50 - Vila das Acácias | CEP $12.228-904$ São José dos Campos/SP _ Brazil | Email: humbaman@uol.com.br

Received: $27 / 06 / 13$ | Accepted: $23 / 10 / 13$ 


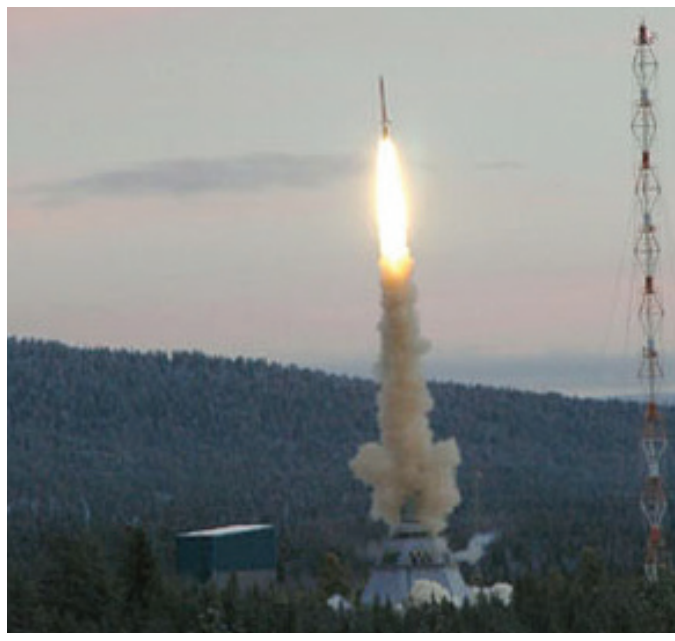

Figure 2. VSB-30 takes off from Esrange, Sweden. reaches the speed of 7,500 km/h $(2,100 \mathrm{~m} / \mathrm{s})$ while still flying within the atmosphere. For practical purposes the dense part of the atmosphere is assumed to be $90 \mathrm{~km}$ thick. Beyond $90 \mathrm{~km}$, vacuum conditions are assumed.

As a result of such very high speeds, aerodynamic heating arises as a major problem in the VSB-30 design. This problem is larger near the stagnation regions, such as those existing at the nose cap and the fins leading edges, where temperature surpasses $2,100^{\circ} \mathrm{C}$ (Machado and Pessoa-Filho, 2007). Indeed, it is mandatory to obtain an accurate evaluation of the reached heat flux and the temperatures, in order to correct size the thermal protection. Along the years ablative materials have been effectively used as TPS of space vehicles. This is the case of the nose cap of VSB-30, where a composite material, like Si-phenolic resin, is used (Da Costa et al., 1996; Tick et al., 1965), Fig. 4.

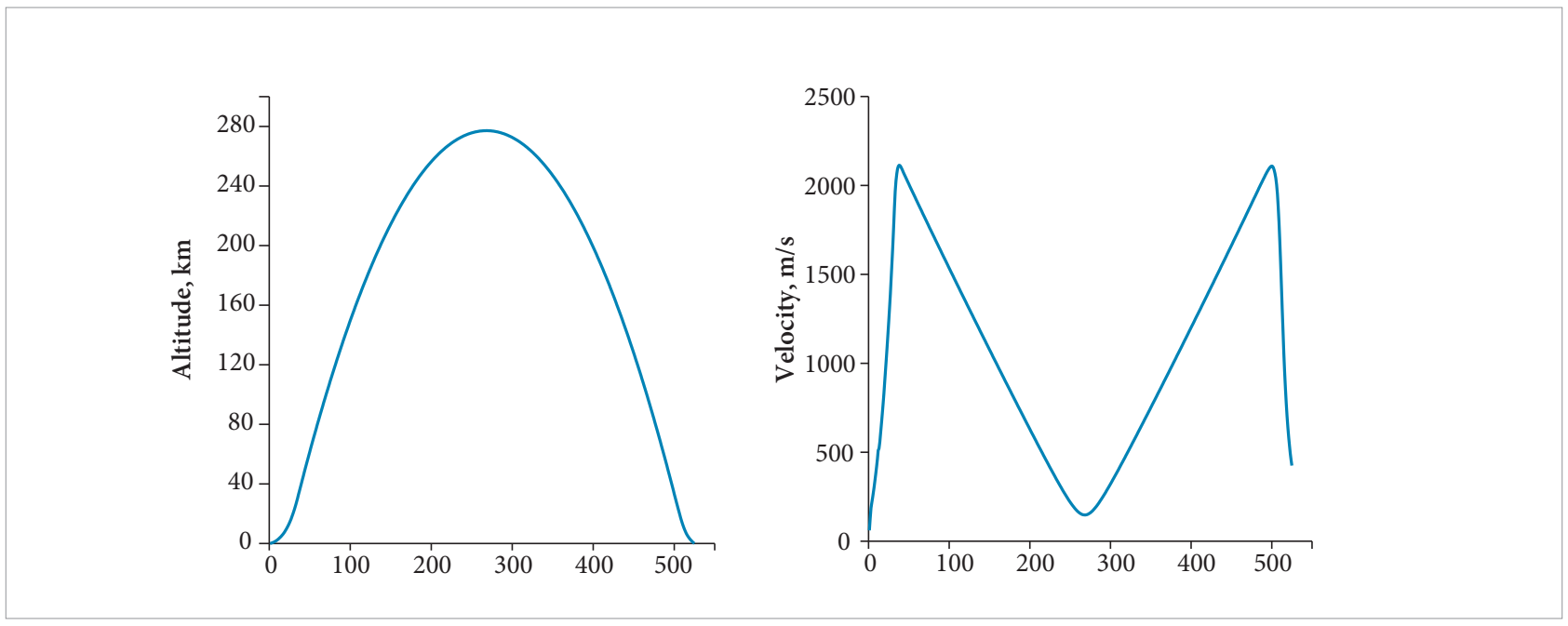

Figure 3. VSB-30 altitude-velocity maps.
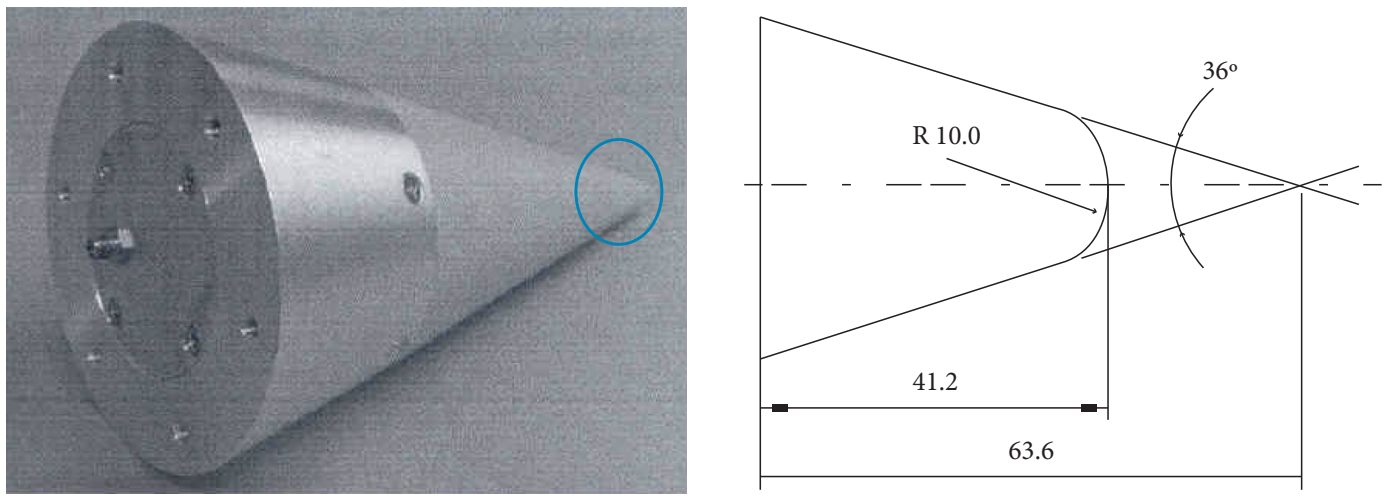

Figure 4. VSB-30 nose cap. 
The conventional ablation model considers one front and treats ablation as single phase change process at constant temperature. In this work a model for the ablative process with two moving fronts (char-melting and pyrolysis) is applied in the computational simulation of the ablation in the thermal shield (TPS) of the VSB-30 sounding rocket, in the vicinity of the stagnation point. The objective is to compare the results of the two models, in order to evaluate the difference in design parameters, like external temperature and surface variation.

This work is an extension of the previous work of Machado (2012a), in which the validation of the two-fronts model and the numerical method for ablation can be found. In that work, the two-fronts model has been already compared with some experimental results, presenting a much better agreement than the one-front model. Such procedure will allow a more accurate dimensioning of the rocket thermal protection system, contributing for project optimization.

The interface tracking method proposed by Unverdi and Tryggvason (1992) was employed to solve the moving front problem. This method is based in a hybrid EulerianLagragean approach that presents the advantage of using a regular homogenous mesh and represents the interface as accurate as more points are added to the grid. The equations of mass and energy conservation in their differential form are used to represent the thermal and ablative behavior of the heat shield with the time related to local temperature and thickness variations and solved through the finite volume method.

In the following sections, the physical problem and the method of solution are described, and the final results for the ablation are presented.

\section{PHYSICAL PROBLEM AND MATHEMATICAL MODEL}

\section{AERODYNAMIC HEATING}

To predict the heat transfer on VSB-30, it is necessary to know the pressure, temperature and velocity fields around the rocket. That can be accomplished by numerically solving the boundary layer equations. However, such a procedure is expensive and time consuming. In the present work a simpler, but reliable, engineering approach is used, which allows to obtain the convective heat transfer coefficient and the representative environment temperature for heat exchange without needing of a numerical solution of the boundary layer equations. The following simplifying assumptions are made:

- zero angle of attack;

- the rotation around its longitudinal axis is neglected;

- atmospheric air is considered to behave as a calorically and thermally perfect gas (no chemical reactions).

Although those hypotheses are not exact, they provide a good approximation to the real situation for the case of a ballistic reentry and for VSB-30 flight in particular, as shown by Machado (2012b). Such hypotheses should be revised in the case of planetary reentry.

The free stream conditions ahead of the nose cap are those given by $v_{\infty}, T_{\infty}, p_{\infty}$, corresponding, respectively, to velocity, temperature and pressure. By knowing $v_{\infty}$ and altitude, as function of time, together with an atmospheric model (U.S. Standard Atmosphere, 1976), it is possible to evaluate the free stream properties. For supersonic flow $\left(M_{\infty}>1\right)$, a bow shock wave appears ahead of the nose. By using the normal shock relationships (Anderson Jr., 1989), it is possible to calculate $v_{1}, T_{1}$ and $p_{1}$ after the shock, in the centerline.

The heat flux over the external surface was calculated through the Zoby's method (Zoby et al., 1981; Miranda and Mayall, 2001). This method employs the heat flux calculation at the stagnation point, obtained through the method of Cohen, as a starting point to integrate the temperature distribution inside the thermal boundary layer over the external surface. That distribution is computed by relating heat transfer to a skin-friction relation based on the momentum thickness through a modified Reynolds analogy form. Corrections for the compressibility (using the Eckert's reference enthalpy relation) and turbulence are added to the calculation. The velocity, temperature and pressure outside the boundary layer are assumed to be that estimated after the shock. The method can be employed in constant and variable entropy-edge conditions and both, reacting and non-reacting gas mixtures. Details of the solution can be found in the work of Machado (2008). Using the Zoby's method, the convective heat transfer coefficient, $H$, is calculated along the $y$-coordinate that is measured along the body's surface: $y=0$ corresponds to the stagnation point, and $R$ is a geometric parameter shown in Fig. 5, in which the red line represents the nose cap surface.

It should be pointed out that such a procedure is performed along the nose cap's surface (following the $y$-coordinate), for different trajectory times. Therefore, $H=H(y, t)$. The variations of the convective heat transfer coefficient and the adiabatic wall 
temperature $\left(T_{a w}\right)$, also called recovery temperature $\left(T_{r}\right)$, at stagnation point are shown in Fig. 6. An energy balance at the surface, accounting the radiative heat transfer, provides the heat absorbed by the wall.

\section{HEAT CONDUCTION AND ABLATION}

Once the convection heat transfer and the adiabatic wall temperature are known, wall temperature distributions can be determined solving the heat transfer equations. VSB-30 nose cap is covered with a composite material (Si-Phenolic resin), which works as an ablative TPS. Until the ablation temperature is reached, a transient heat conduction process

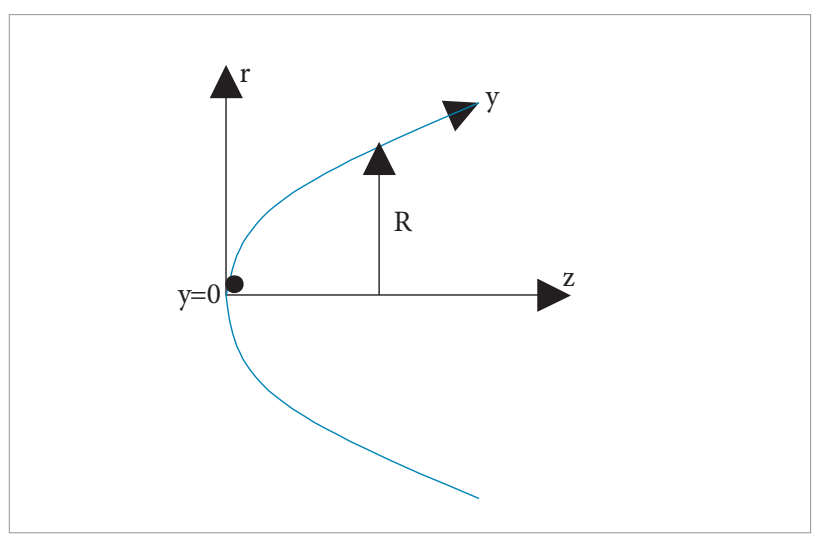

Figure 5. Coordinate system.

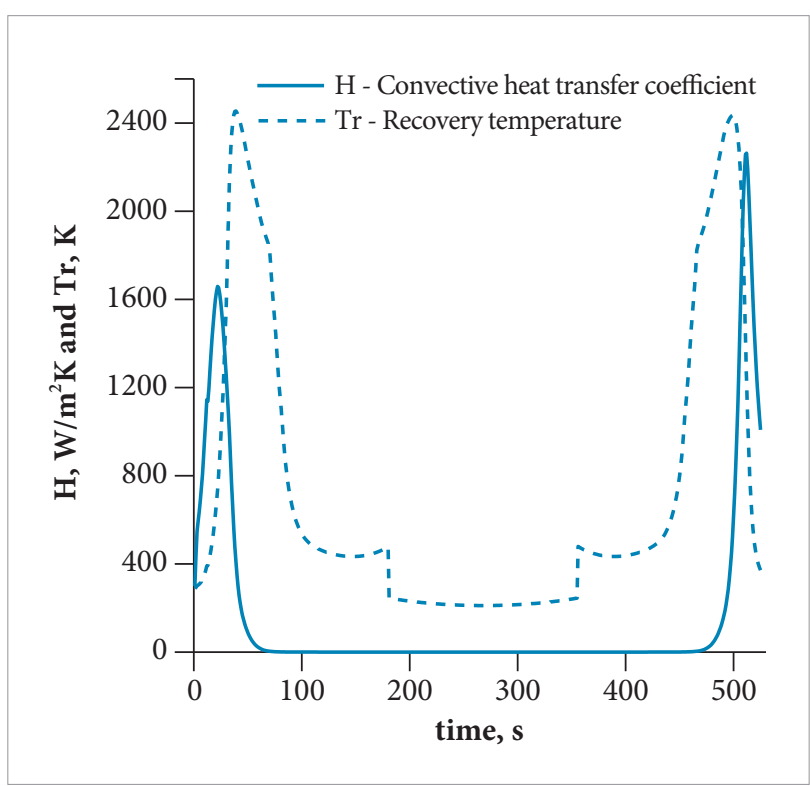

Figure 6. Recovery temperature and convective heat transfer coefficient at stagnation point, obtained through the Zoby's Method, during VSB-30 trajectory. occurs. Once the TPS surface reaches the ablation temperature, its thickness is reduced; therefore, a transient, coupled conduction, moving boundary problem appears. In this work, the ablation model proposed for a composite material will consider the presence of two layers: the virgin material and the char layer that appears after the pyrolysis front. Two moving fronts will be accounted for: the pyrolysis front and the ablation front of the char layer, both starting at constant temperatures.

The set of equations used to represent the physical problem is written according to the interface tracking method (Juric, 1996). The nose cap and the surrounding airflow are represented as parts of a continuous domain of calculation. The application of the energy conservation principle to an infinitesimal volume element in the mathematical domain leads to a partial differential equation for the temperature, namely:

$\frac{\partial\left(\rho \cdot C_{p} \cdot T\right)}{\partial t}=\nabla(K . \nabla T)+Q$

where $\rho$ is the density, $C_{p}$ is the specific heat, $T$ is the temperature, $t$ is the time, $K$ is the thermal conductivity and $Q$ is a source term that accounts for the net heat exchange at the fronts, which will be mathematically represented as interfaces:

$Q=\int_{A} q \delta\left(\boldsymbol{x}-\boldsymbol{x}_{F}\right) d A$

where $x$ is the position in the coordinate system, $x_{F}$ is the interface position, $A$ is the area, and $q$ is the source term of energy per unit of surface of the interface and must be adapted to the physical model used to represent the behavior of each interface. The following hypothesis will be assumed to build the mathematical model for the ablative and heat conduction processes in the structure:

- solid materials are considered isotropic with constant properties;

- the pyrolisis zone is considered a zero thickness front. Pyrolisis enthalpy and temperature are considered constant;

- the char layer recession occurs through oxidation or sublimation, at constant temperature. The aerodynamic removing of material is neglected;

- absence of melting layer;

- full reaction of the gases and perfect mixing with the air in the boundary layer around the external surface, with negligible influence over the air physical properties;

- $\quad$ air is treated as an ideal gas; 
- the flow field around the surface is not affected by the change in the surface geometry and gas injection;

- radiation is absorbed or emitted for surface, but not transmitted.

All these assumptions have being used in previous studies (Machado, 2012a), with successful results in representing the physical process. The second assumption, in particular, was studied by Sias (2009), which has concluded it was accurate enough, when compared to more complex models for ablation. All the chemistry occurring in the ablative process is implicit and is included in the ablation parameters (heat and temperature of pyrolysis). Although the hypothesis of perfect gas behavior for air might yield inaccurate results for the recovery temperature, it occurs in altitudes where the convective heat transfer coefficient approaches to zero, resulting in a negligible heat flux over the surface, and should not affect the ablation. According to these hypothesis, the heat balance in the external surface yields:

$q=\rho L V+H(t, y)\left[T_{F}\left(t, x_{F}\right)-T_{a w}\right]+\mathcal{E} \sigma\left[T_{F}^{4}\left(t, x_{F}\right)-T_{a w}^{4}\right]$

where $V$ is the interface velocity, $L$ is the heat of ablation of the char layer, $H$ is the convection heat transfer coefficient, $T_{F}$ is the interface temperature and $T_{a w}$ is the adiabatic wall temperature, also called recovery temperature of the air, $\varepsilon$ is the emissivity and $\sigma$ is the Boltzman constant. One should note that this term might exist in every moving interface. In the pyrolisis front it is simplified, once there is no convection to or from the external flow and the radiative heat transfer is supposed to not occur between the layers (since there is no transmission):

$q=\rho L_{p} V$

In this case, $L_{p}$ is the heat of pyrolisis. The flux of injection gases is also neglected due its low specific mass, when compared to the solid material. It is remarkable that the specific mass that appears in Eqs. 3 and 4 is the interface specific mass.

Although the airflow is included in the domain, its effects are implicit in the convection coefficient $H$. As a consequence, this region is considered adiabatic, and the heat capacity and thermal conductivity are assumed to be null. Once ablation temperature $\left(T_{A}\right)$ is reached, the interface condition becomes:

$T_{F}-T_{A}=0$
A similar jump condition appears in the pyrolisis front (the pyrolisis temperature, $T_{p}$, replacing $T_{A}$ ).

\section{METHOD OF SOLUTION}

The moving boundary problem was solved by the Interface Tracking Method, introduced by Unverdi and Tryggvason (1992), and employed by Juric (1996) in the solution of phase change problems. In this method, a fixed uniform Eulerian grid is generated, where the conservation laws are applied over the complete domain. The interface acts as a Lagragean referential, where a moving grid is applied. The instantaneous placement of the interface occurs through the constant remeshing of the moving grid, and each region of the domain is characterized by the Indicator Function, which identifies the properties of the wall and the air around it.

This method allows for the representation of any geometry used in the TPS, and also the characterization of every layer separately. It is accomplished without a high increase in the computational cost and does not need any pre-processing (construction of unstructured grid or coordinate transformation). In this work, this method is employed to estimate the ablative performance of the TPS, considering a two-dimensional approach in both, the heat conduction and the moving boundary problem. Although here an axisymmetric simulation is performed, the method can be applied to any 2-D geometry. The method is detailed described in the previous work of Machado (2012a), and will be briefly summarized as follows.

The interface is represented as a parametric curve, $\mathrm{R}(u)$, where the normal and tangent vectors and curvature are extracted from. The interface points are interpolated by a $4^{\text {th }}$ order Lagrange polynomial that uses two points before and two after the point to be interpolated, which allows one to analytically obtain the geometric parameters and remeshes the curve, keeping the distance $d$ between curve points within the interval $0.9<d / h<1.1$, where $h$ is the distance among the fixed grid points, as shown in Fig. 7 .

The phase distribution in the domain of calculation is represented with the Indicator Function, $I(x, t)$. For the simple case of only two phases, it varies from 0 (air) to 1 (solid), and it is numerically constructed using the interface curve to determine a source term $\mathrm{G}(x)$. The jump of the indicator function across the interface is distributed over the fixed grid points, yielding a gradient field in the mesh: 
$G(x)=\nabla I=\int_{A} n \delta\left(x-x_{f}\right) d A$

where $n$ is the normal unitary vector, and the term $\delta\left(x-x_{f}\right)$ should be zero, except over the interface, as represented by the Dirac delta function, $\delta$. However, such a representation is not convenient for a discrete number of points, since the Lagrangian points, $x_{k}$, do not necessarily coincide with the Eulerian grid points, $x_{i, j}$. The Distribution Function is used to represent the interface discontinuity as a continuous and smooth function. Such a function is similar to a Gaussian distribution function and its value depends on the distance $\left|x_{i j}-x_{k}\right|$ between the Lagrangean and Eulerian points:

$D_{i, j}\left(\boldsymbol{x}_{k}\right)=\frac{f\left[\left(x_{k}-x_{i}\right) / h\right] \cdot f\left[\left(y_{k}-y_{j}\right) / h\right]}{h^{2}}$

where $D_{i j}$ is the Distribution Function for a point $k$ in the Lagrangean mesh with respect to a Eulerian point $\left(x_{i} y_{j}\right)$. One should note that increasing $h$ results in a thicker interface. The function $f$ is the probability distribution related to the distance $h$ as:

$f(x)= \begin{cases}f_{1}(x) & \text { if }|x| \leq 1 \\ 1 / 2-f_{1}(2-|x|) & \text { if } 1<|x|<2 \\ 0 & \text { if }|x| \geq 2\end{cases}$

$f_{1}(x)=\frac{3-2|x|+\sqrt{1+4|x|-4 x^{2}}}{8}$

The divergence of the gradient field is found by numerical derivation of Poison's equation:

$\nabla^{2} I=\nabla . G$

Despite being considered constants in each phase, the properties inside the domain must be treated as variable in the formulation. A generic property $\phi(\rho, C p$ or $K)$ is expressed as:

$\phi(x)=\phi_{0}+\left(\phi_{1}-\phi_{0}\right) I(x, t)$

where $f_{0}$ and $f_{1}$ are the property values for phases 0 and 1 (according to their values of indicator function), respectively.

The coupling between the moving mesh and the fixed grid is done at each time step, through the Distribution Function, used to represent the source terms in the balance equations and to interpolate the infinitesimal discontinuities into a finite

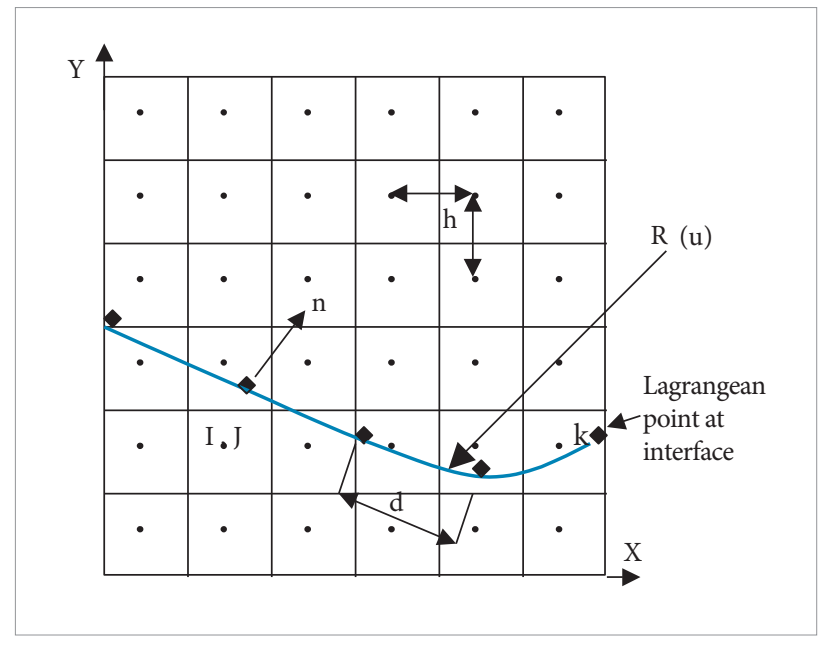

Figure 7. Eulerian and Lagrangean meshes.

thick region at the interface. In a similar manner, this function is used to interpolate the field variables from the Eulerian grid to the interface. The equations used to distribute the source term in the field and interpolate variables to the interface are:

$Q_{i, j}=\sum_{k} q_{k} D_{i, j}\left(x_{k}\right) \cdot \Delta s_{k}$

$T_{k}=\sum_{i, j} h^{2} T_{i, j} D_{i, j}\left(x_{k}\right)$

where $D s_{k}$ is the average of the straight line distances from the point $k$ to the two points on either side of $x_{k}$, and corresponds to the area concerned to the point $x_{k}$ in the interface surface. Equation 11 is the dicretized form of Eq. 2, where the Dirac delta function was replaced by the distribution function, $D_{i, j}$, which is also done in Eq. 6.

The initial interface shape, $R(u)$, is first specified and then the Indicator Function is constructed. From the initial conditions, the property and temperature fields are determined. Out of the ablative period, the interface temperature keeps bellow the ablation temperature, and the energy equation is solved as a pure heat conduction problem, via the Finite Volume Method, employing the well-known FTCS discretization in an explicit time marching schedule (Patankar, 1980).

As the interface reaches the ablation temperature at a given point, an iterative process starts up, in order to determine the interface velocity at each time step, which must satisfy the temperature condition, Eq. 5, at that interface point. The process goes on as far as the point temperature is equal to ablation temperature. The steps to be followed are: 
1. using the current value of interface velocity, $V^{n}$ (result from the previous time step), the interface points are transported to a new position, calculated explicitly through the equation $V^{n}=\left(d x_{f} / d t\right) . \mathrm{n}$, where $\mathrm{n}$ is normal unitary vector;

2. density and specific heat are calculated at the new interface position;

3. $V^{n+1}$ is estimated via Newton iterations, using a numerical relaxation schedule, Eq. 13;

4. heat flux $q$ crossing the interface is calculated through Eqs. 3 or 4, depending on the interface, and distributed into the fixed grid through Eq. 11;

5. according to the boundary conditions, energy equation, Eq. 1, is used to obtain the temperature at time step $n+1$;

6. temperature is interpolated to find $T_{F}$ at the interface, using Eq. 12;

7. the temperature jump condition is tested and if it is lower than the reached tolerance, the fields of viscosity and conductivity are updated for the new position, and one step in time is advanced. If that is not the case, a new estimate for $V^{n+1}$ is calculated and the process returns to step 5 .

The convergence criterion used in step 7 is the residual in Eq. 5. Once it has reached the desired tolerance, convergence for interface velocity is assumed. Otherwise, the velocity is corrected via Newton Iterations, given as:

$V^{n+1}=V^{n}-\omega \cdot R(T)$

where $\omega$ is a relaxation factor and $R(T)$ is the residual for the temperature jump condition at the interface. Iterations are repeated until $R(T)$ in every point become smaller than the prescribed tolerance. The optimum value for $\omega$ is found by numerical experimentation, at the beginning of the calculation. The method was compared with the analytical solution for a simple phase change problem resulting in an excellent agreement (Ruperti Jr., 1991).

In the case of more than one interface, an Indicator Function, $I_{m}$, is created for each interface, in order to characterize every region concerned to the interfaces individually. Therefore, in a region $m$ (that corresponds to a specific phase or material), a generic property is estimated as:

$\phi=\sum_{m=1}^{N F C} \phi_{m} I g_{m}$ where NFC is the number of interfaces. The Lagrangean grids for all interfaces have the same values for the parameters $h$ and $d$, shown in Fig. 1, and are constructed from a particular parametric curve $R_{m}(u) . I g_{\mathrm{i}}$ is the Global Indicator Function for a region $m$, obtained from the Indicator Function of each interface (calculated as described before). It is given as:

$I g_{m}=I_{m-1}-I_{m}$

$I_{0}=1 ; I_{N F C}=0$

If there are more than one moving interface, the source term $Q_{m}$ for every interface has to be extracted from a modified form of Eq. 2:

$Q_{m}=\sum_{k} \int_{A} q_{m} \delta_{m}\left(x-x_{F m, k}\right) d A_{m}$

Actually, according to the numerical method, Eq. 11 will be used to calculate the source term in every interface. The total amount of heat generated will be the summation of the heat sources of all interfaces:

$Q=\sum_{m} Q_{m}$

The convergence criterion and velocity correction are the same as those for the case of one interface, but they are extended to all interfaces at each time step.

\section{RESULTS}

The results were obtained for the region near the stagnation point of VSB-30, Fig. 8, which corresponds to a circular semisphere with radius of $280 \mathrm{~mm}$. Note that the $Y$-coordinate has a different meaning of $y$-coordinate shown in Fig. $3(y)$. Since the flight is considered with zero angle of attack, the problem is considered to be axy-symmetric, and only the half of that region has to be simulated. A 20x20 points grid over a domain of $12 \times 12 \mathrm{~mm}$ was employed to simulate the heat transfer and moving boundary problem, with a tolerance of $10^{-6}$ for the residual in Eq. 5. A resulting 26 points Lagrangean mesh was obtained for the interface used to represent the external surface.

The convergence of the domain representation by the Indicator Function is shown in Fig. 9, for a cutting view of the domain in the diagonal starting from the axes origin. 
The more points are added to the mesh the more the thickness reduces. When the number of points reaches $20 \times 20$, the interface representation is assumed to be satisfactory, since the rise of the processing time would not compensate the correspondent reduction of interface thickness.

Figure 10 shows the final interface position according with the number of mesh points used, where the results for $15 \times 15$ and 20x20 meshes are very close. In Fig. 11, the variation of external temperature with time at the stagnation point shows an even better convergence between these two meshes. Considering these results, the $20 \times 20$ mesh was considered refined enough for the purposes of this study.

The results were compared with those from the one-front model (Machado, 2008), using the same numerical parameters. Due to the difference between the models, the composite material (Si-phenoic resin) was characterized in a different way. The properties of resin employed in each model are shown in Table 1.

Figure 12 shows the characterization of every region in the domain through the global indicator function, at $t=0$. In this case, the two interfaces corresponding to the pyrolisis and carbonization fronts are placed at the same initial position, over the external surface, since the ablative process did not start yet. The colored region corresponds to the interfaces and do not represent exactly the discontinuity, presenting a slope and a finite thickness (about $0.6 \mathrm{~mm}$ ). This thickness could be reduced through the increase of the number of grid points, which was not considered necessary, according to the convergence analysis.

Figure 13 shows the 3 -D view of temperature distribution at various moments. Temperature peaks occur in the external surface, in direct contact with the heated air, during the ascension,

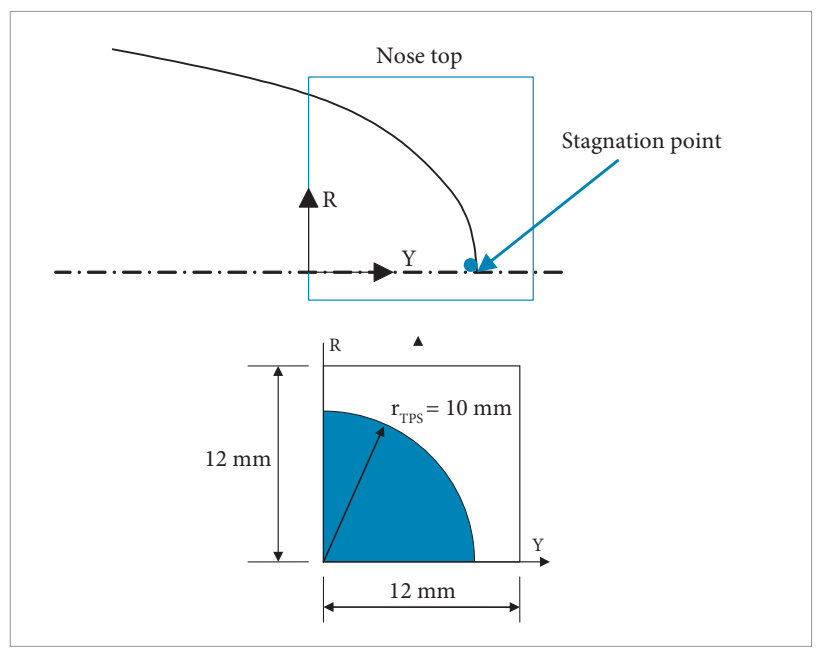

Figure 8. Domain of calculation, regions (TPS in blue) and dimensions. at 35 seconds (Fig. 13a) and at 500 seconds during the reentry (Fig. 13c). Between these times, at 100 seconds (Fig. 13b) and after the reentry heating, at 524 seconds (Fig. 13d), a surface cooling occurs. In this case, the external surface becomes a heat sink and yields a "valley" in the temperature profile. One can observe that the temperature of fusion of the char is not reached. Indeed, this surface does not move, since there is no phase change and only the pyrolysis front moves when the pyrolisis temperature of the resin is reached. The region correspondent to the air does not present relevant temperature changes, once it is considered to be adiabatic with zero thermal capacity. Temperature distribution and the external surface position correspondent to these profiles are shown in Fig. 14.

The results were compared to those obtained through the one layer model, where ablation is treated as single-phase change process. Figure 15 shows temperature of internal and external surfaces, both at $R=0$, along the symmetry axis (at $Y=10 \mathrm{~mm}$ and $Y=0$, respectively), what corresponds to the stagnation point line. The two temperature peaks correspond to the ascension and reentry of the vehicle in the atmosphere, including a period between then where a cooling occurs, due the heat losses by radiation, in the absence of convection ( $H=0$, according to Fig. 4). It is noticeable that the ablative process begins when the external surface reaches the pyrolisis temperature, and the ablation front starts to move. The temperature of fusion of the char is not reached at both peaks, even in the points of maximum temperature. For the one layer model, the temperature of ablation is lower, what means the process begins earlier and takes more time. Due the presence of the char layer in the two-fronts model, the temperatures reached in the internal surfaces are lower, what

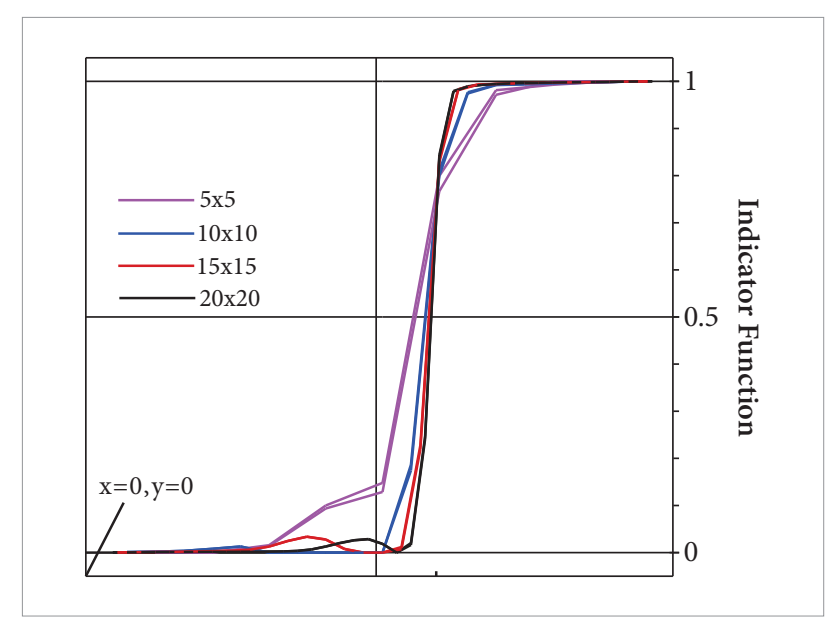

Figure 9. Indicator Function profile observed in the diagonal trough the axes origin, for diverse number of points used in the regular mesh. 


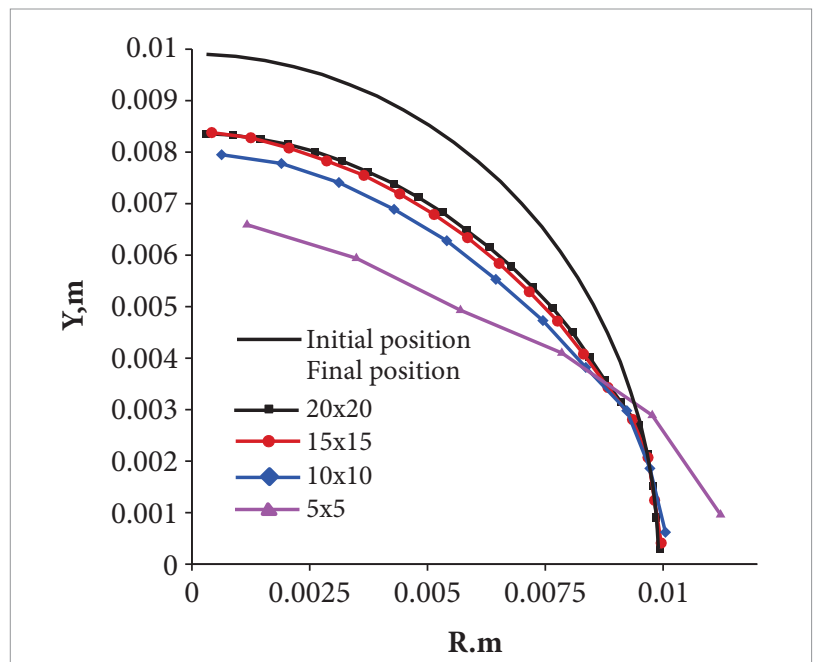

Figure 10. Interface position for the virgin material at the final time, for diverse number of points used in the regular mesh.

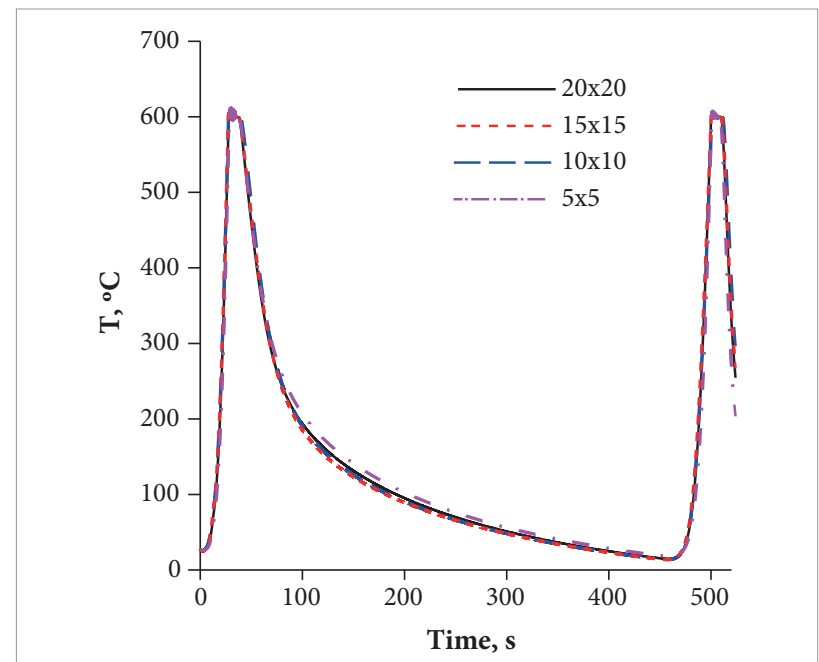

Figure 11. External surface temperature with time, for diverse number of points used in the regular mesh.

Table 1. Properties of Si-phenolic resin.

\begin{tabular}{|c|c|c|c|}
\hline \multirow{2}{*}{ Property } & \multirow{2}{*}{ One-front model } & \multicolumn{2}{|c|}{ Two-front model } \\
\hline & & Virgin material & Char \\
\hline Thermal conductivity $\left(\mathrm{W} / \mathrm{m}^{\circ} \mathrm{C}\right)$ & $0.485^{(1)}$ & $0.485^{(1)}$ & $0.428^{(2)}$ \\
\hline Specific heat, $\mathrm{Cp}\left(\mathrm{J} / \mathrm{kg}{ }^{\circ} \mathrm{C}\right)$ & $1,256^{(1)}$ & $1,256^{(1)}$ & $879.5^{(2)}$ \\
\hline Specific mass, $\rho\left(\mathrm{kg} / \mathrm{m}^{3}\right)$ & $1,730^{(1)}$ & $1,730^{(1)}$ & $1,300^{(2)}$ \\
\hline Emissivity, $\varepsilon$ & $0.8^{(1)}$ & $0.8^{(1)}$ & $0.8^{(3)}$ \\
\hline Heat of ablation (MJ/kg) & $12^{(4)}$ & - & - \\
\hline Temperature of ablation $\left({ }^{\circ} \mathrm{C}\right)$ & $538^{(4)}$ & - & - \\
\hline Heat of pyrolisis $(\mathrm{MJ} / \mathrm{kg})$ & - & $0.78^{(5)}$ & - \\
\hline Temperature of pyrolisis $\left({ }^{\circ} \mathrm{C}\right)$ & - & $599^{(5)}$ & - \\
\hline Heat of fusion/Sublimation (MJ/kg) & - & - & $10.5^{(3)}$ \\
\hline Temperature of fusion $\left({ }^{\circ} \mathrm{C}\right)$ & - & - & $3,700^{(3)}$ \\
\hline
\end{tabular}

(1)Da Costa et al. (1996); (4)Gregori et al. (2008); (5)Tick et al. (1965); (2)Williams and Curry (1992); (3)Savvatimskii (2003).
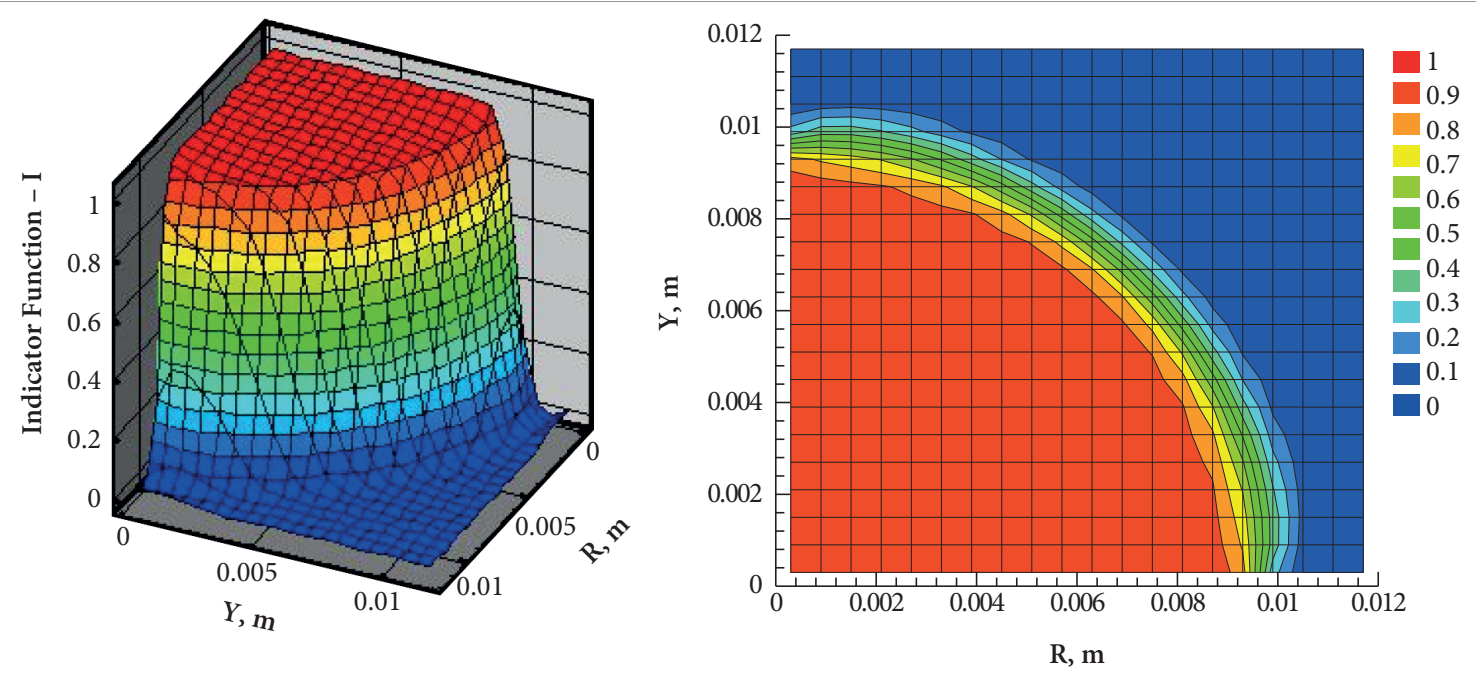

Figure 12. Global Indicator Function for every region: Thermal Protection System in red and air in blue. 


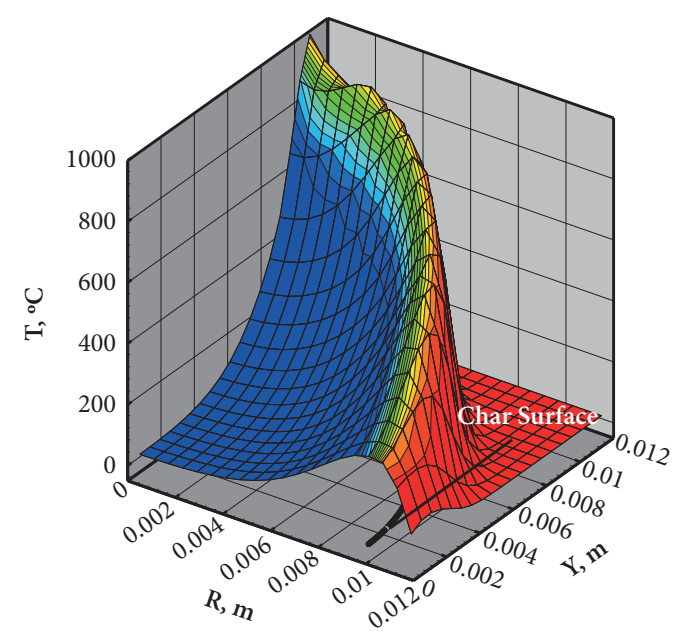

(a) $\mathrm{t}=35 \mathrm{~s}$

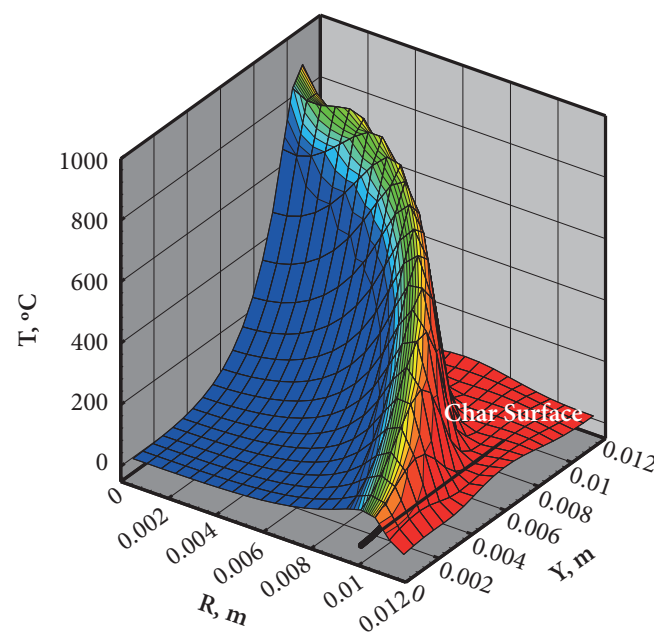

(c) $t=500 \mathrm{~s}$

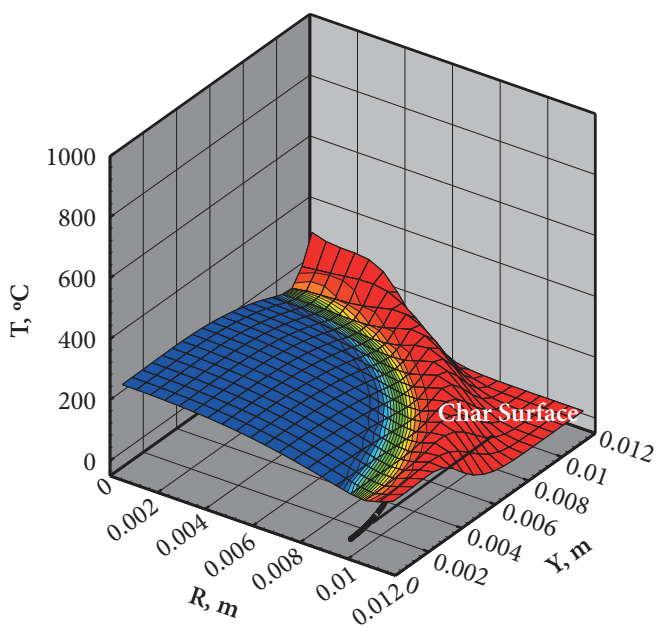

(b) $\mathrm{t}=100 \mathrm{~s}$

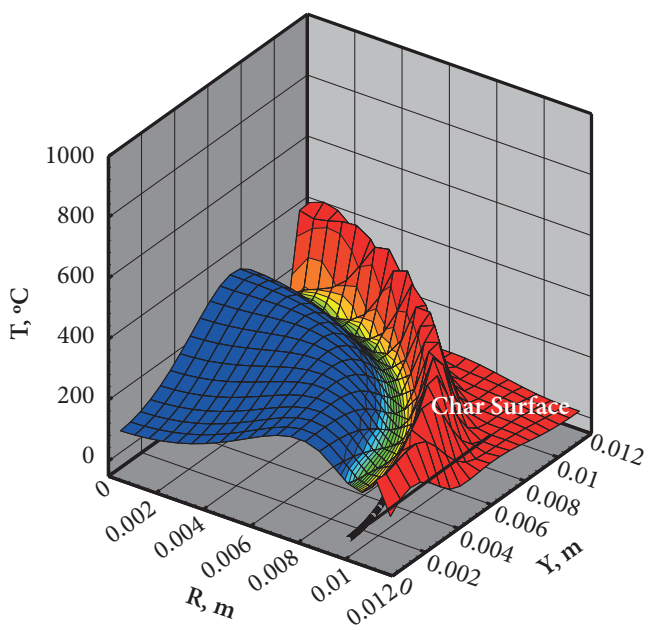

(d) $t=524 s$

Figure 13. 3-D view of temperature field during the trajectory, with the Global Indicator Function for every region: Thermal Protection System in blue and air in red.

indicates that this layer works as a thermal barrier for the virgin material of the TPS.

Figure 16 shows the interface position with the time, after the two ablation periods (ascension and reentry). According to the results, the ablation is more intense closer the stagnation point for both models. However, the two-fronts model indicates a lower material consumption than the one layer model. The initial position of the external surface keeps constant in the twofronts model, since the temperature of fusion/sublimation of the char is not reached. The displacement starts after the formation of the pyrolisis front (considered to be initially at $0.1 \mathrm{~mm}$ after the external surface, for calculation purposes).

\section{CONCLUSION}

In this work, the two-dimensional transient aerodynamic heating and ablation processes in the vicinity of the stagnation point of the VSB-30 sounding rocket TPS were simulated through an interface tracking method considering the presence of two moving fronts, the pyrolisis and the carbonization fronts, and two resulting layers, the virgin material and the char layer. Preliminary results demonstrated that the method is able to capture the temperature peaks and to represent the ablation process as a moving boundary problem, in the presence of more 


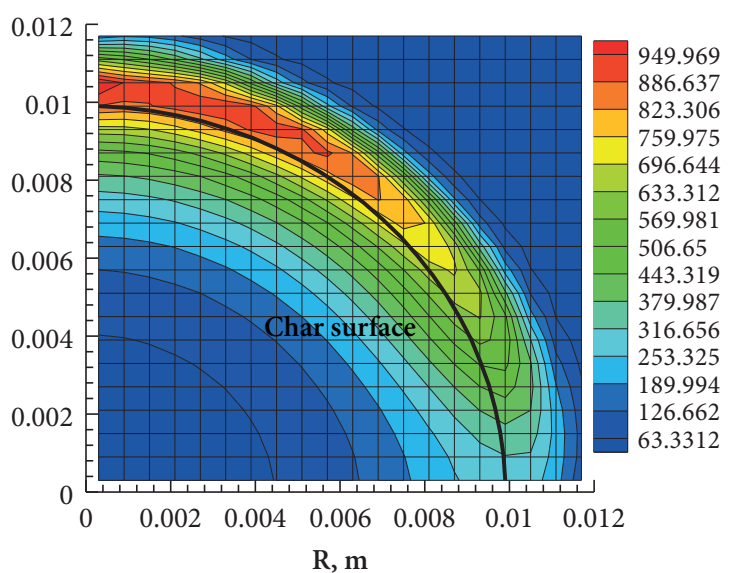

(a) $\mathrm{t}=35 \mathrm{~s}$

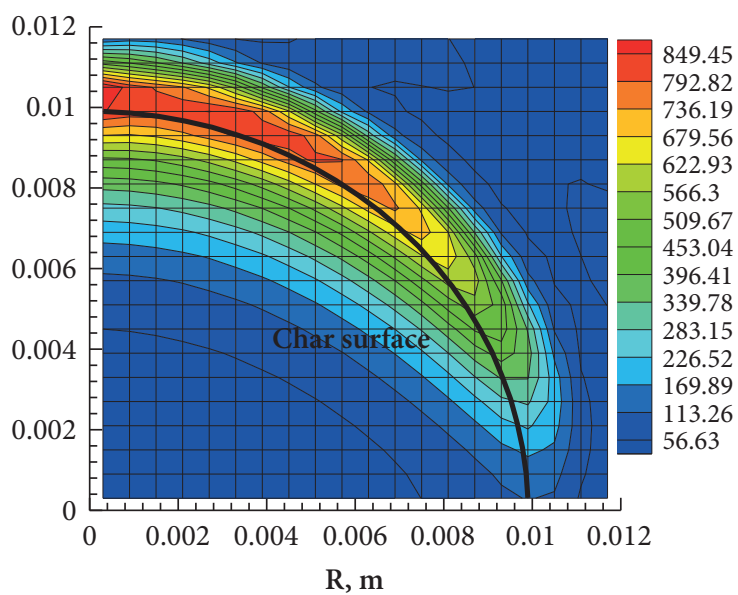

(c) $\mathrm{t}=500 \mathrm{~s}$

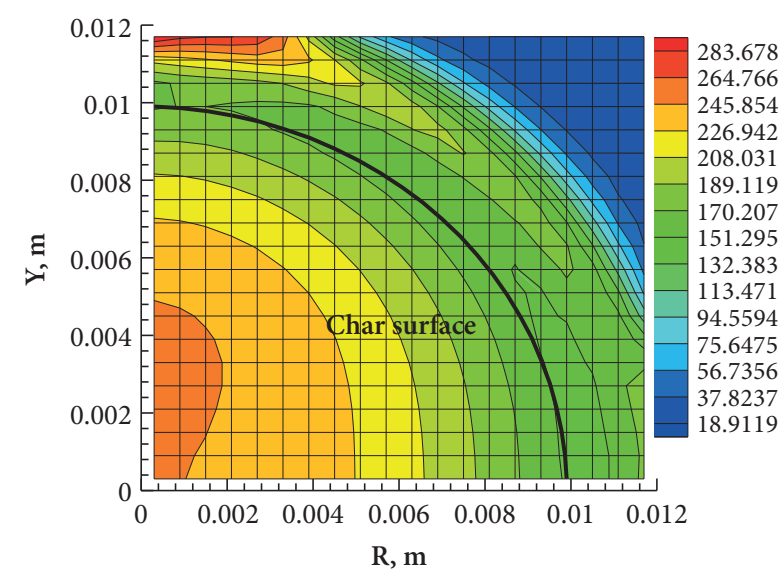

(b) $t=100 s$

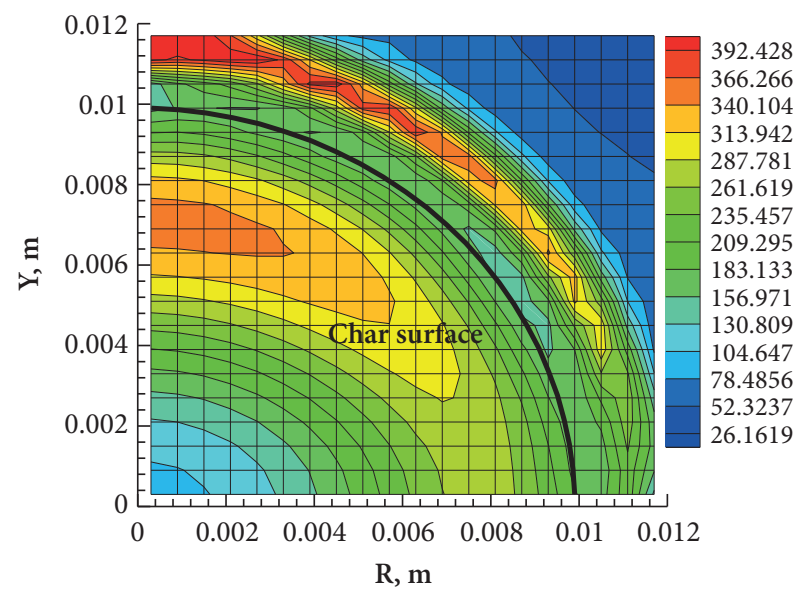

(d) $t=524 s$

Figure 14. Temperature distribution during the trajectory.

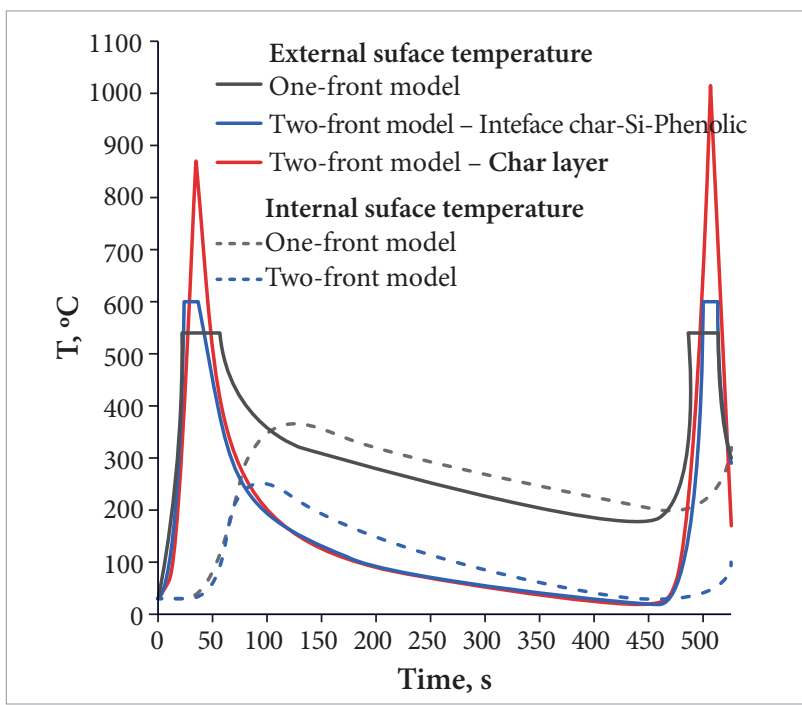

Figure 15. Comparison between the results of the ablation models for temperatures in the stagnation point, $R=0$.

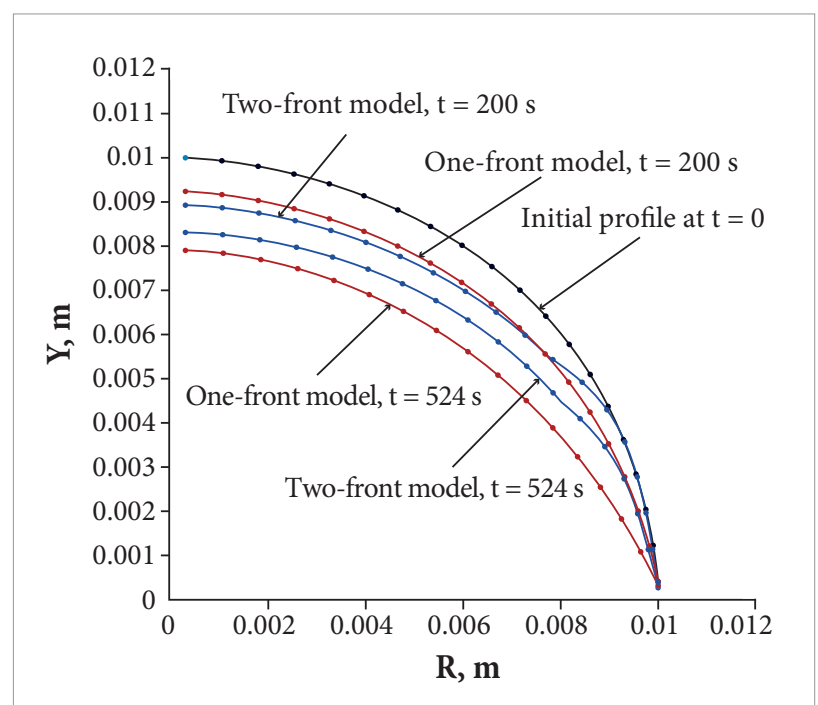

Figure 16. Comparison between the results of the ablation models for interface position with time. 
than a single moving front, which allows representing diverse physical simultaneous processes.

When compared with the one-front model, it results in lower temperatures out of the periods of heating, shorter periods of ablation and less consumption of protective material. This analysis can be extended to more regions of the rocket, more layers and other shapes, including more moving fronts, if it is necessary. A more realistic physical model for the ablation in the composite material may now replace the one used in this work. The inclusion of the flow field effects, like injection of mass due to sublimation, shall also be incorporated into the simulation.

Since the two-fronts model has presented better accuracy than the one-front model, as shown in previous studies already mentioned, it seems to be a better option to estimate TPS performance for other vehicles and might provide a more accurate dimensioning and consequently reducing its weight and cost.

\section{ACKNOWLEDGMENTS}

The author would like to thank the Conselho Nacional de Desenvolvimento Científico e Tecnológico (CNPq), the Brazilian Federal Agency for Science and Technology, for the financial support during this work.

\section{REFERENCES}

Anderson Jr., J.D., 1989, "Hypersonic and High Temperature Gas Dynamics", McGraw-Hill, Blacklick, Ohio, USA.

Da Costa, L.E.V.L., De Mello, F.C. and Pardini, L.C., 1996, "Viability Study of Thermal Protection for SARA Platform", IAE/CTA, Technical note NT-130ASE-N/96, IAE/CTA, São José dos Campos, São Paulo, Brazil (in Portuguese).

Garcia, A., Yamanaka, S.S.C., Barbosa, A.N., Bizarria, F.C.P., Jung, W. and Sheuerpflug, F, 2011, "VSB-30 Sounding Rocket: History of Flight Performance", Journal of Aerospace Technology and Management, Vol. 3, No.3, pp. 325-330. doi: 10.5028/JATM.2011.03032211

Gregori, M.L., Barros, E. de A., Petraconi Filho, G., Costa, S.F. and Pardini, L.C., 2008, "Properties of Quartz-Phenolic Composites for Thermal Protection Systems", 59 th IAC Congress, Glascow, Scotland.

Juric, D., 1996, "Computations of Phase Change," Ph.D. thesis, University of Michigan, Michigan, $166 \mathrm{p}$.

Machado, H.A. and Pessoa-Filho, J.B., 2007 , "Aerodynamic Heating on VSB-30", ESA Symposium, Visby, Sweden

Machado, H.A., 2008, "Two-dimensional Simulation of Ablation due to Aerodynamic Heating in a Sounding Rocket", 40 ${ }^{\text {th }}$ AIAA Thermophysics Conference, Seattle, USA.

Machado, H.A., 2012a, "Simulation of Ablation in a Composite Thermal Protection System via an Interface Tracking Method", Journal of Aerospace Technology and Management, Vol. 4, No. 3, pp. 331340. doi: 10.5028/jatm.2012.04030312

Machado, H.A., 2012b, "After-Flight Thermal Analysis of the VSB30 Sounding Rocket Payload", VII National Congress of Mechanical Engineering - CONEM, São Luís, Maranhão, Brazil (in Portuguese).

Miranda, I.F. and Mayall, M.C de M., 2001, "Fluxo de Calor Convectivo em Micro-Satélites em Reentrada atmosférica”, Graduate Dissertation, Instituto Tecnológico de Aeronáutica, São José dos Campos, São Paulo, Brazil, 66 p.
Patankar, S.V., 1980, "Numerical Heat Transfer and Fluid Flow", Taylor \& Francis, New Delhi.

Ruperti Jr., N.J., 1991, "Solution of a One-Dimensional Ablation Model", Master thesis, Instituto Nacional de Pesquisas Espaciais, São José dos Campos, São Paulo, Brazil (in Portuguese).

Savvatimskii, A. V., 2003, "Melting Point of Graphite and Liquid Carbon", Physics-Uspekhi, Vol. 46, No. 12, pp. 1295-1303. doi:10.1070/ PU2003v046n12ABEH001699

Sias, D.F., 2009, "Hibrid Solutions for Heat Transfer on Ablative Thermal Protection Systems", Master thesis, Universidade Federal do Rio de Janeiro, Rio de Janeiro, Brazil (in Portuguese).

Tick, S.J., Huson, G.R. and Griese, R., 1965, "Design of Ablative Thrust Chambers and Their Materials", Journal of Spacecraft and Rocket, Vol. 2, No. 3, pp. 325-331. doi: 10.2514/3.28179

Turner, J., Hörschgen, M., Turner, P., Ettl, J., Jung, W. and Stamminger, A., 2005, "SHEFEX - The Vehicle and Sb-Systems for a Hypersonic Re-entry Flight Experiment", 17th ESA Symposium in European Rocket and Balloon Programmes and Related Research, Sandfjord, Norway.

U.S. Standard Atmosphere, Washington, D.C., U.S. Government Printing Office, 1976.

Unverdi, S.O. and Tryggvason, G., 1992, "A Front-Tracking Method for Viscous, Incompressible, Multi-fluid Flows", Journal of Computational Physics, Vol. 100, No. 1, pp. 25-37.

Williams, S.D. and Curry, D.M., 1992, "Thermal Protection Materials Thermophysical Property Data”, NASA Reference Publication 1289.

Zoby, E.V., Moss, J.N. and Sutton, K., 1981, "Approximate Convective Heat Equations Hypersonic Flows", Journal of Spacecraft and Rockets, Vol. 18, No. 1, pp. 64-70. 